TITLE:

\title{
Effect of relative humidity on heat transfer across the surface of an evaporating water droplet in air flow
}

AUTHOR(S):

KUROSE, Ryoichi; FUJITA, Akitoshi; KOMORI, Satoru

CITATION:

KUROSE, Ryoichi ... [et al]. Effect of relative humidity on heat transfer across the surface of an evaporating water droplet in air flow. Journal of Fluid Mechanics 2009, 624: 57-67

ISSUE DATE:

2009-04

URL:

http://hdl.handle.net/2433/109955

RIGHT:

Copyright (C) Cambridge University Press 2009 


\title{
Effect of relative humidity on heat transfer across the surface of an evaporating water droplet in air flow
}

\author{
RYOICHI KUROSE†, AKITOSHI FUJITA \\ AND SATORU KOMORI \\ Department of Mechanical Engineering and Science and \\ Advanced Research Institute of Fluid Science and Engineering, Kyoto University, \\ Yoshida-honmachi, Sakyo-ku, Kyoto 606-8501, Japan
}

(Received 12 September 2008 and in revised form 26 December 2008)

A three-dimensional direct numerical simulation (DNS) is applied to flows inside and outside an evaporating spherical water droplet in air, and the effect of relative humidity on the heat transfer between the droplet and ambient air is investigated. The initial air temperature is set to be $15 \mathrm{~K}$ higher than the initial droplet temperature. The results show that the local evaporation heat loss indicates the maximum on the front of the droplet and decreases on going from the front to the rear, and the reduction on the rear becomes marked for high-droplet Reynolds numbers. This is because the evaporation rate is suppressed on the rear surface by the presence of flow separations behind the droplet. The droplet temperature decreases in the low-humidity condition, whereas it increases in the high-humidity condition. This difference is caused by the heat balance of evaporation heat loss and convective heat gain from ambient air at the droplet surface.

\section{Introduction}

Dispersed water droplets are often seen in environmental air flows in rain, cloud, mist, sea spray and so on. It is therefore of great importance to precisely estimate heat and mass transfer between water droplets and atmospheric air in developing a reliable climate model.

Kurose et al. (2003) and Bagchi \& Kottam (2008) applied three-dimensional direct numerical simulations (DNS) to flows around a sphere, and investigated mass or heat transfer from a sphere to ambient flows. However, since they assumed the sphere to be rigid and neglected the flow inside the sphere, simple conditions that mass concentration and temperature in the sphere are uniform and constant were given. On the other hand, Sugioka \& Komori $(2005,2007)$ recently performed a DNS of flows both inside and outside a sphere, and studied the flow interaction and mass $\left(\mathrm{CO}_{2}\right)$ transfer from air to a water droplet across the droplet surface. They showed that the mass absorbed from the droplet surface is diffused inside the droplet by internal circulations and that the mass flux decreases with time. Nevertheless, the heat transfer between a water droplet and air has not been discussed yet. Concerning the heat transfer, heat loss due to droplet evaporation is considered to play an important role. 


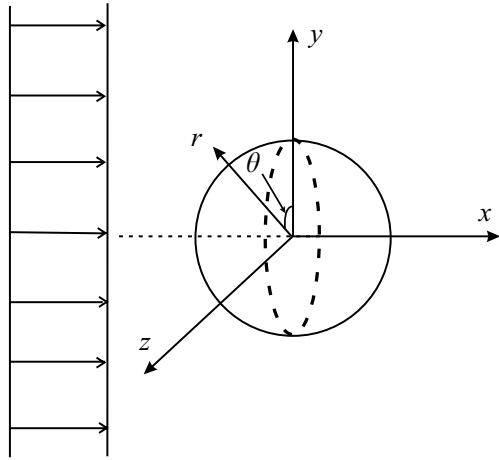

Figure 1. Coordinate system for a droplet in a uniform flow.

The purpose of this study is therefore to investigate the heat transfer across the surface of an evaporating water droplet in air flow by applying a DNS to flows both inside and outside the droplet. Since the evaporation rate is considered to be dominated by the relative humidity of ambient air, the effect of the relative humidity is carefully discussed.

\section{Direct numerical simulation}

2.1. Flow configuration and governing equations

The flow geometry and coordinate system for the computations of flows inside and outside an evaporating droplet are shown in figure 1. The ambient flow around the droplet is a uniform gaseous flow. In this study, the deformation of the droplet from the spherical shape, the thermocapillary effects and the droplet movement in response to the hydrodynamic force are neglected. The decrease in the droplet diameter and the outflow velocity from the droplet surface (Kurose et al. 2003) owing to the evaporation are also neglected. The governing equations are three-dimensional continuity, NavierStokes (NS), vapour conservation (mole fraction) and thermal energy equations in cylindrical coordinates given in non-dimensional forms based on the mean free stream velocity $U_{c}$ and the diameter of the droplet $d$ by

$$
\begin{aligned}
\nabla \cdot \boldsymbol{U} & =0, \\
\frac{\partial U_{x}}{\partial t}+(\boldsymbol{U} \cdot \nabla) U_{x} & =-\frac{\partial p}{\partial x}+\frac{1}{R e_{d, k}} \nabla^{2} U_{x}, \\
\frac{\partial U_{r}}{\partial t}+(\boldsymbol{U} \cdot \nabla) U_{r}-\frac{U_{\theta}^{2}}{r} & =-\frac{\partial p}{\partial r}+\frac{1}{R e_{d, k}}\left(\nabla^{2} U_{r}-\frac{U_{r}}{r^{2}}-\frac{2}{r^{2}} \frac{\partial U_{\theta}}{\partial \theta}\right), \\
\frac{\partial U_{\theta}}{\partial t}+(\boldsymbol{U} \cdot \nabla) U_{\theta}+\frac{U_{r} U_{\theta}}{r} & =-\frac{1}{r} \frac{\partial p}{\partial \theta}+\frac{1}{R e_{d, k}}\left(\nabla^{2} U_{\theta}-\frac{U_{\theta}}{r^{2}}+\frac{2}{r^{2}} \frac{\partial U_{r}}{\partial \theta}\right), \\
\frac{\partial C}{\partial t}+(\boldsymbol{U} \cdot \nabla) C & =\frac{1}{R e_{d, k} S c_{k}} \nabla^{2} C, \\
\frac{\partial \Theta}{\partial t}+(\boldsymbol{U} \cdot \nabla) \Theta & =\frac{1}{R e_{d, k} \operatorname{Pr}_{k}} \nabla^{2} \Theta,
\end{aligned}
$$

where $\boldsymbol{U}\left(=\left(U_{x}, U_{r}, U_{\theta}\right)\right)$ is the fluid velocity, $C$ the vapour mole fraction, $\Theta$ the temperature and $p$ the pressure. The droplet Reynolds number $R_{d, k}$, the Schmidt 
Effect of relative humidity on heat transfer across an evaporating water droplet 59 number $S c_{k}$ and the Prandtl number $P r_{k}$ are defined as

$$
R e_{d, k}=\frac{\rho_{k} U_{c} d}{\mu_{k}}, \quad S c_{k}=\frac{\mu_{k}}{\rho_{k} D}, \quad \operatorname{Pr}_{k}=\frac{\mu_{k}}{\rho_{k} \alpha_{k}},
$$

respectively. Here $\rho$ is the density, $\mu$ the viscosity, $D$ the molecular diffusivity and $\alpha$ the thermal diffusivity. The subscript $k$ stands for the value inside or outside the droplet by $l$ or $g$ ( $C$ is solved only for the flow outside the droplet in this study). Hereafter $R e_{d, g}, S c_{g}$ and $P r_{g}$ are simply written as $R e_{d}, S c$ and $P r$, respectively. The non-dimensional vapour mole fraction $C$ and temperature $\Theta$ are defined as

$$
\begin{aligned}
& C=\frac{C_{v}-C_{v, \infty}}{C_{v, s}^{0}-C_{v, \infty}}, \\
& \Theta=\frac{T-T_{s}^{0}}{T_{g, \infty}-T_{s}^{0}},
\end{aligned}
$$

where $C_{v}$ and $T$ are the dimensional vapour mole fraction and temperature, respectively. The subscripts $s$ and $\infty$ represent the value at the droplet surface and that of the ambient gas in the far-field conditions, respectively, and the superscript 0 means the initial value. The value at the centre of the droplet $(r=0)$ is given by averaging the values around the centre, because of the singularity in the cylindrical coordinates.

\subsection{Boundary conditions}

The inlet boundary condition of the velocity is given by

$$
U_{x}=1, \quad U_{r}=U_{\theta}=0,
$$

and the outlet boundary condition of the velocity is given by

$$
\frac{\partial \boldsymbol{U}}{\partial x}=0
$$

The velocity boundary conditions on the surface of the droplet are given such that the normal velocity is zero and the tangential velocity and stress are matched at the interface.

In this study, the evaporation rate is very low and, therefore, the vapour concentration at the air-water interface is assumed to be continuous (Onishi 1990). The vapour concentration at the droplet surface is saturated and given by using Teten's formulation (Murray 1967). On the other hand, the temperature boundary condition on the surface of the droplet is given in a non-dimensional form by

$$
\begin{aligned}
\left.\frac{\partial \Theta_{g}}{\partial r}\right|_{\text {surface }} & =\left.A \frac{\partial \Theta_{l}}{\partial r}\right|_{\text {surface }}-\left.B \frac{\partial C}{\partial r}\right|_{\text {surface }}, \\
A & =\frac{\lambda_{l}}{\lambda_{g}}, \\
B & =\frac{D_{v} L_{v}}{\lambda_{g}} \frac{\left(C_{v, s}^{0}-C_{v, \infty}\right) W_{l}}{T_{g, \infty}-T_{s}^{0}} .
\end{aligned}
$$

Here, the second term on the right-hand side of (2.12) represents the heat loss due to droplet evaporation. $\lambda$ is the thermal conductivity, $L_{v}$ the latent heat of vaporization and $D_{v}$ the molecular diffusivity of vapour. The operator $\left.\frac{\partial}{\partial r}\right|_{\text {surface }}$ represents the partial derivative in the normal direction at the droplet surface in spherical coordinates, and 
(a)

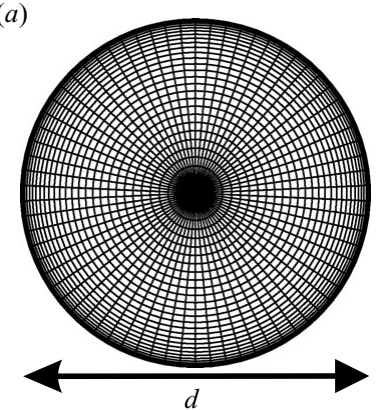

(b)

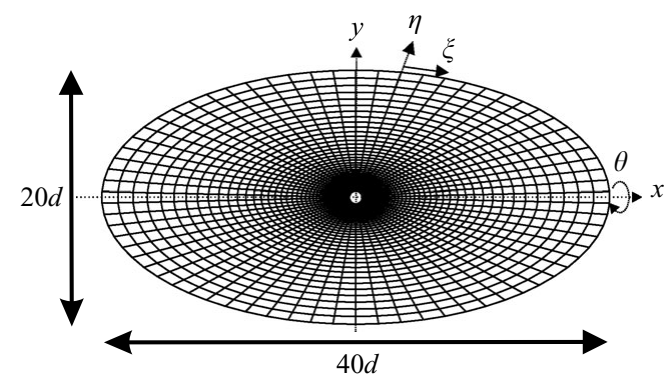

FIGURE 2. Computational domain and grids: $(a)$ inside a droplet and $(b)$ outside a droplet.

the subscript $l$ or $g$ stands for the value inside (liquid) or outside (gas) the droplet. Here, $W_{l}$ is the molecular weight of the vapour.

\subsection{Numerical conditions}

The NS equations were solved directly using a finite-difference scheme based on the marker and cell (MAC) method. The numerical procedure used here was first developed by Hanazaki (1998) and is essentially the same as those used in previous papers (Kurose \& Komori 1999; Kurose, Misumi \& Komori 2001; Kurose et al. 2003; Sugioka \& Komori 2005, 2007). The $(x, r, \theta)$-coordinate system was transferred to the $(\eta, \xi, \theta)$-coordinate system with an equal spacing, and the transformed governing equations were discretized to construct the finite-difference formulation. Numerical grids outside and inside a spherical droplet are shown in figure 2 . The size of the computational domain non-dimensionalized by droplet diameter $d$ were 40 and 20 diameters in the $x$-and $y$-directions, respectively. The grid points inside and outside the droplet used were respectively $35 \times 31 \times 48$ and $35 \times 61 \times 48$ in the $\eta$, $\xi$ and $\theta$ directions.

The fluids inside and outside the droplet are water and air. Their temperatures were set to be $285 \mathrm{~K}$ and $300 \mathrm{~K}$, respectively. The transport properties of water and air required in the computations were obtained in Welty et al. (1984), and those for the mixture were interpolated with respect to their mass fractions. The Schmidt and Prandtl numbers inside and outside the droplet at $300 \mathrm{~K}$ were $S c_{g}(=S c)=0.62$, $\operatorname{Pr}_{l}=6.9$ and $\operatorname{Pr}_{g}(=\operatorname{Pr})=0.70$.

The evaporation and heat transfer were initiated after the computations were almost at the fully developed state. The computations were performed for the droplet Reynolds numbers $\left(R e_{d}\right)$ of 10,50 and 400 and the relative humidities $(H u)$ of 0 and 0.3 at the temperature under consideration. Here, $H u$ is the ratio of the vapour mass to the saturated vapour mass at the temperature. The values of $A$ and $B$ defined by (2.13) and (2.14) were $2.3 \times 10^{1}$ and $-1.7 \times 10^{-3}$ for $H u=0$ and $2.3 \times 10^{1}$ and $-4.7 \times 10^{-4}$ for $H u=0.3$, respectively.

In this study, the capillary number $\left(=\mu_{g} U_{c} / \sigma\right.$, where $\sigma$ is the surface tension $)$ and the Weber number $\left(=\rho_{g} U_{c}^{2} d / \sigma\right)$ of the water droplet in the air flow were $1.2 \times 10^{-3}$ and 0.48 at maximum, respectively (Sugioka \& Komori 2007). Also, it was estimated that the decrease ratio of the droplet diameter is approximately $5.6 \%$ (i.e. eventual droplet diameter/initial droplet diameter $=94.4 \%$ ) and the non-dimensional outflow velocity from the droplet surface based on $U_{c}$ is $2.2 \times 10^{-11}$ at maximum. Therefore, it is considered that the droplet deformation and the size reduction are negligibly small. 
Effect of relative humidity on heat transfer across an evaporating water droplet 61

(a)

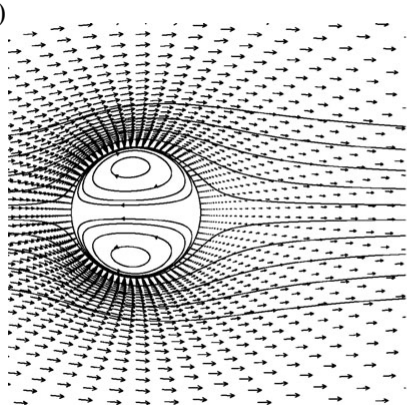

(b)

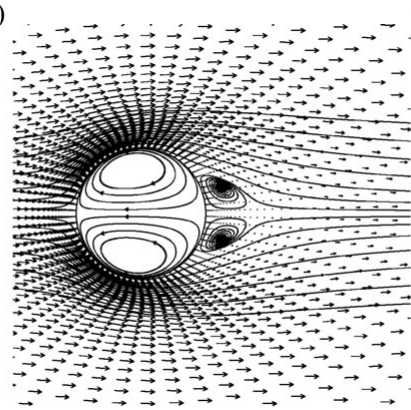

(c)

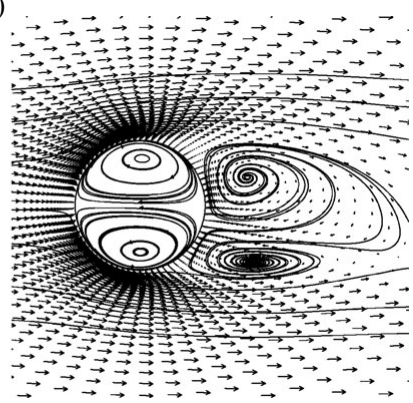

FIGURE 3. Instantaneous velocity vectors and streamlines inside and outside a droplet on the $x-y$ plane: (a) $R e_{d}=10,(b) R e_{d}=50$ and (c) $R e_{d}=400$.

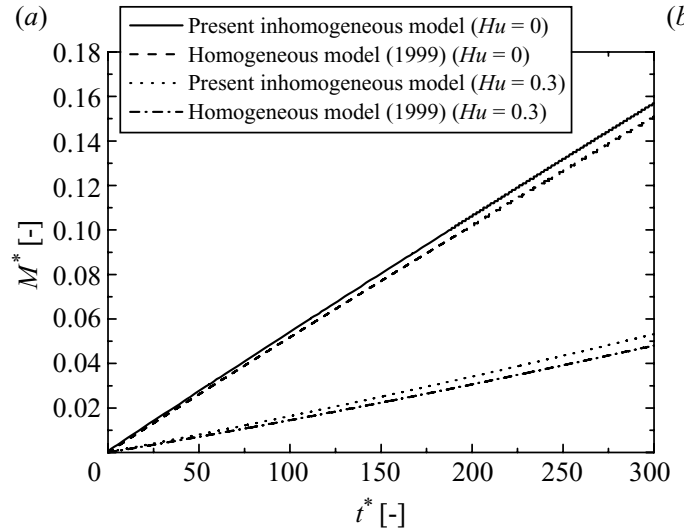

(b)

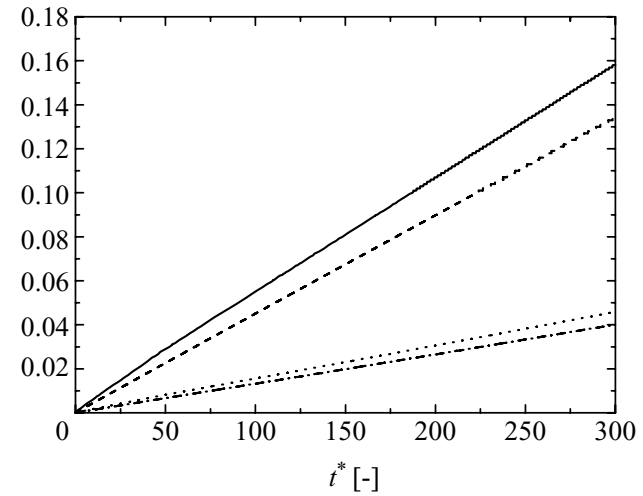

FIGURE 4. Effect of relative humidity $H u$ on the time variation of the total amount of evaporated mass $M^{*}:(a) R e_{d}=10$ and $(b) R e_{d}=400$.

\section{Results and discussion}

\subsection{Flow field and droplet evaporation rate}

Figure 3 shows the velocity vectors and streamlines inside and outside a droplet on the $x-y$ plane at $R e_{d}=10,50$ and 400 . It is found that the flow fields inside and outside the droplet are weakly asymmetric with respect to the $x$-axis for both $R e_{d}=10$ and 50 , whereas the flow field outside the droplet is strongly asymmetric for $R e_{d}=400$ due to the vortex shedding which occurs behind the droplet. Compared to the flow field outside the droplet, the asymmetry of the flow field is weak inside the droplet. This is because liquid motion inside the droplet does not respond to each gaseous vortex motion with high frequency. The detailed behaviour of these flow fields is discussed in Sugioka \& Komori (2007) and in works by other researchers (e.g. Kim, Elghobashi \& Sirignano 1995; Masoudi \& Sirignano 2000).

The effects of relative humidity $H u$ on the time variation of the total amount of evaporated mass $M^{*}$ at $R e_{d}=10$ and 400 are shown in figure 4 . The values are compared to those obtained by a non-equilibrium Langmuir-Knudsen evaporation model (e.g. Miller \& Bellan 1999; Nakamura et al. 2005; Baba \& Kurose 2008) in which vapour mass fraction and temperature on the droplet surface are assumed to be homogeneous (referred to as homogeneous model hereafter). Here, $M^{*}$ is nondimensionalized by the initial droplet mass. The non-dimensional time $t^{*}$ is defined 


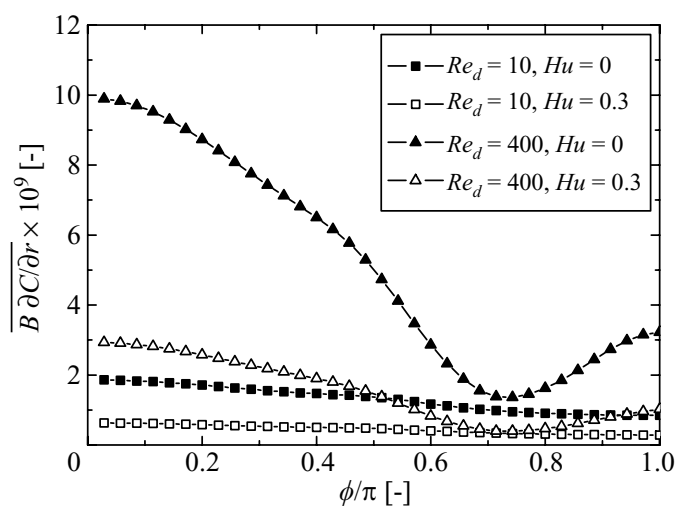

FIGURE 5. Effect of relative humidity $H u$ and droplet Reynolds number $R e_{d}$ on circumferential-averaged local evaporative heat loss $\overline{B \partial C / \partial r}$.

as $t^{*}=R e_{d} \mu_{g} t / \rho_{g} d^{2}$. For both $R e_{d}=10$ and 400 , the present $M^{*}$ linearly increases with time and the rate drastically decreases with increasing $H u$. The similar trends of $M^{*}$ with time and $H u$ are observed using the homogeneous model (Miller \& Bellan 1999). However, the magnitudes of $M^{*}$ by the homogeneous model are consistently lower than those by the present inhomogeneous model (2.12). This suggests that the assumption of the homogeneous vapour mass fraction and temperature on the droplet surface tends to underestimate the droplet evaporation rate, especially for high-droplet Reynolds numbers and low relative humidities.

Figure 5 shows the effect of relative humidity $H u$ and droplet Reynolds number $R e_{d}$ on circumferential-averaged local evaporative heat loss $\overline{B \partial C / \partial r}$ given by the second term on the right-hand side of (2.12). Here, $r$ is the normal component in spherical coordinates. As a whole, $\overline{B \partial C / \partial r}$ tends to increase as $R e_{d}$ increases and $H u$ decreases. It is also found that $\overline{B \partial C / \partial r}$ exhibits a maximum on the front of the droplet and decreases on going from the front to the rear. For high-droplet Reynolds numbers, the reduction of $\overline{B \partial C / \partial r}$ on the rear becomes marked and the minimum appears around $\phi / \pi=0.7$. This may be attributed to the fact that the evaporation rate, which has a linear relation to the evaporative heat loss (see (2.12)), is suppressed on the rear of the droplet surface by the presence of vortices behind the droplet.

To elucidate the relationship between the evaporative heat loss and the vortex shedding, the time variations of instantaneous local evaporative heat loss $B \partial C / \partial r$ along the upper and lower surfaces of a droplet on the $x-y$ plane at $R e_{d}=400$ are shown in figure 6 , together with the corresponding velocity vectors and streamlines outside the droplet on the $x-y$ plane. Here, $r$ is the normal component in spherical coordinates. In the figure, locations indicating the maximum and minimum $B \partial C / \partial r$ on the rear of the droplet are indicated by arrows A-I. It is found that although the maximum and minimum $B \partial C / \partial r$ locations change with time, they are placed in the regions where vortices-oriented reversed flows attach on the droplet surface and the flow separation points exist, respectively. These trends are also observed in figure 7, in which the time variations of distributions of instantaneous local evaporative heat loss $B \partial C / \partial r$ on the rear surface of a droplet are shown. In the figure, the regions where the air velocity in the vicinity of the droplet surface is less than 0.01 are enclosed by black lines. These regions which are considered to contain the flow separation and attached points are found to locate near the maximum and minimum $B \partial C / \partial r$ locations. As described in (2.12), the evaporative heat loss is a linear function of 
Effect of relative humidity on heat transfer across an evaporating water droplet 63

(a)

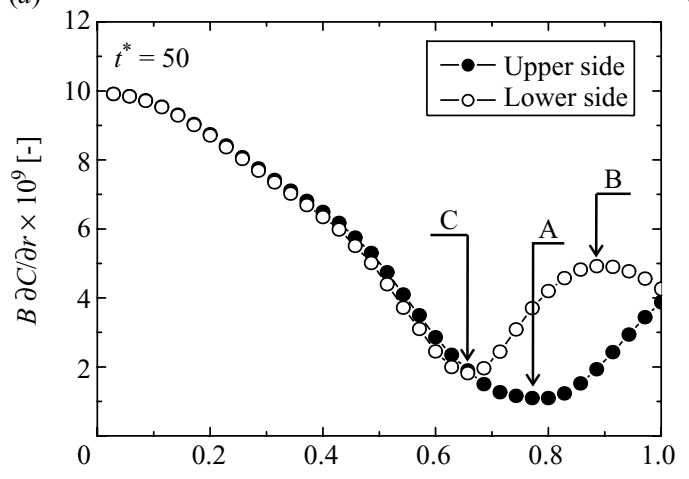

(b)
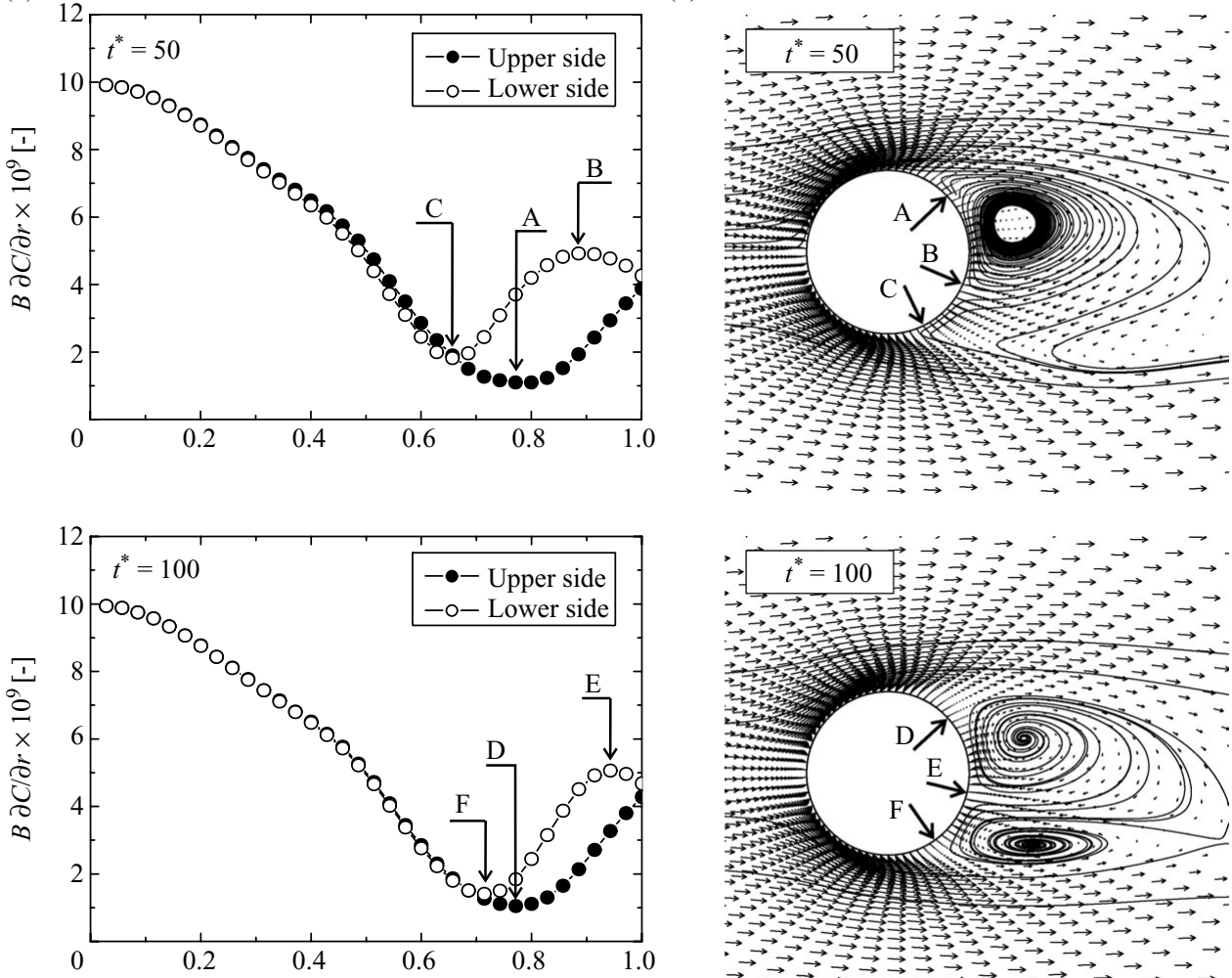

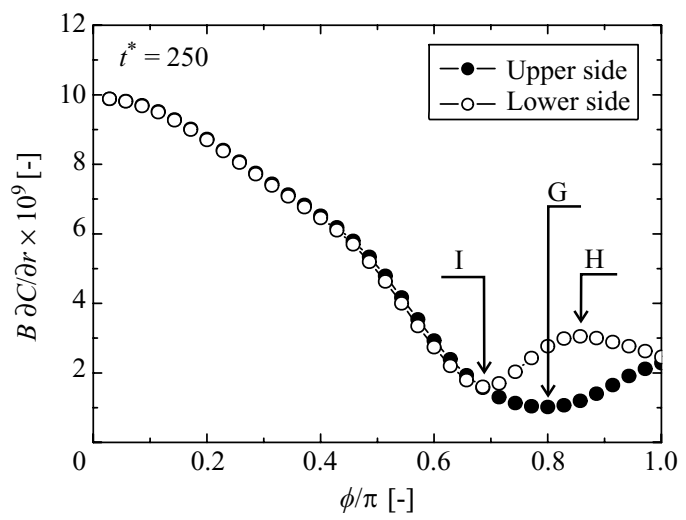

FIGURE 6. Time variations of instantaneous local evaporative heat loss $B \partial C / \partial r$ along the upper and lower surfaces of a droplet on the $x-y$ plane and corresponding velocity vectors and streamlines outside a droplet at $R e_{d}=400$ for $H u=0$ : (a) local evaporative heat loss and (b) velocity vectors and streamlines.

the evaporation rate, which is dependent on the vapour mass fraction gradient in the vicinity of the droplet surface $\partial C / \partial r$. Therefore, it is considered that the flow separations appearing behind the droplet for high-droplet Reynolds numbers make the vapour mass fraction gradient gentle in the vicinity of the surface, which reduces the evaporation rate and then the evaporative heat loss on the rear of the droplet. 

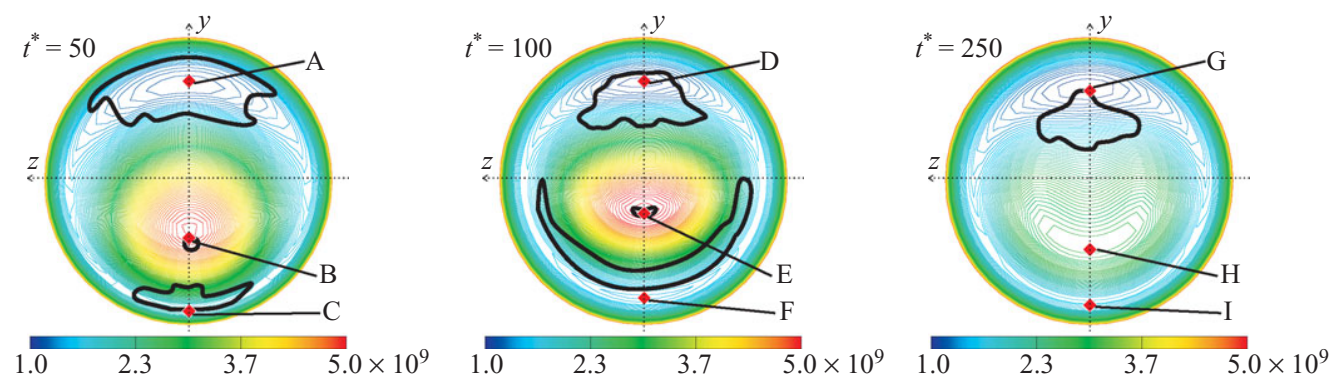

Figure 7. Time variations of distributions of instantaneous local evaporative heat loss $B \partial C / \partial r$ on the rear surface of a droplet at $R e_{d}=400$ for $H u=0$.

(a)
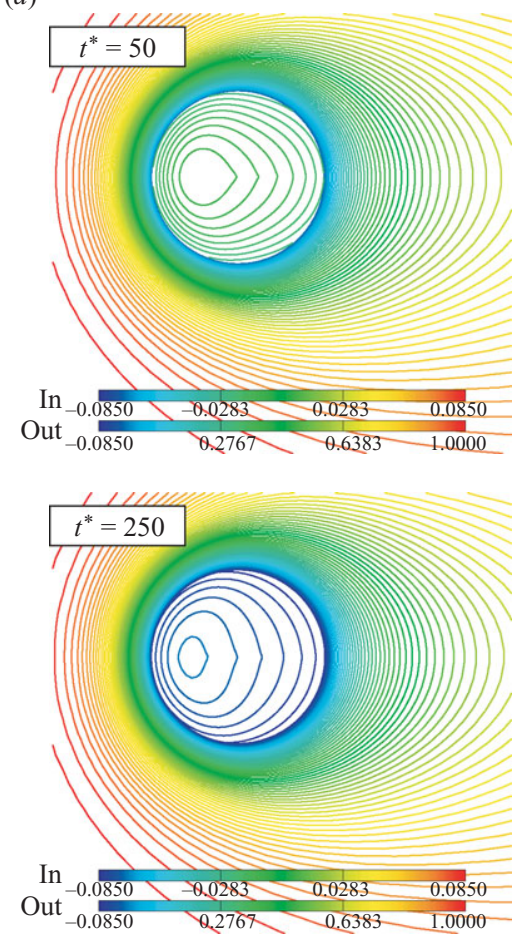

(b)
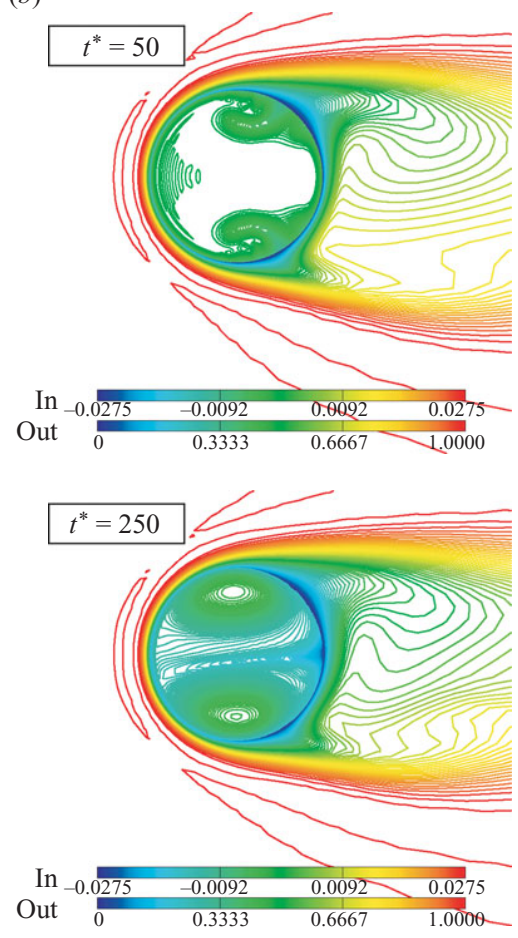

Figure 8. Time variations of distributions of instantaneous temperature $\Theta$ inside and outside a droplet on the $x-y$ plane for $H u=0:(a) R e_{d}=10$ and (b) $R e_{d}=400$.

\subsection{Temperature field}

Figure 8 shows the time variations of distributions of instantaneous temperature $\Theta$ inside and outside a droplet on the $x-y$ plane at $R e_{d}=10$ and 400 for $H u=0$. It is found that the temperatures inside and outside the droplet both decrease with time, and like the flow fields (see figure 3 ) their temperature distributions are weakly asymmetric for $R e_{d}=10$ and strongly asymmetric for $R e_{d}=400$, respectively (it should be noted that cusps on the centreline inside the droplet for $R e_{d}=10$ are drawn due to the commercial visualization software, but this is not realistic). Since 
Effect of relative humidity on heat transfer across an evaporating water droplet 65

(a)
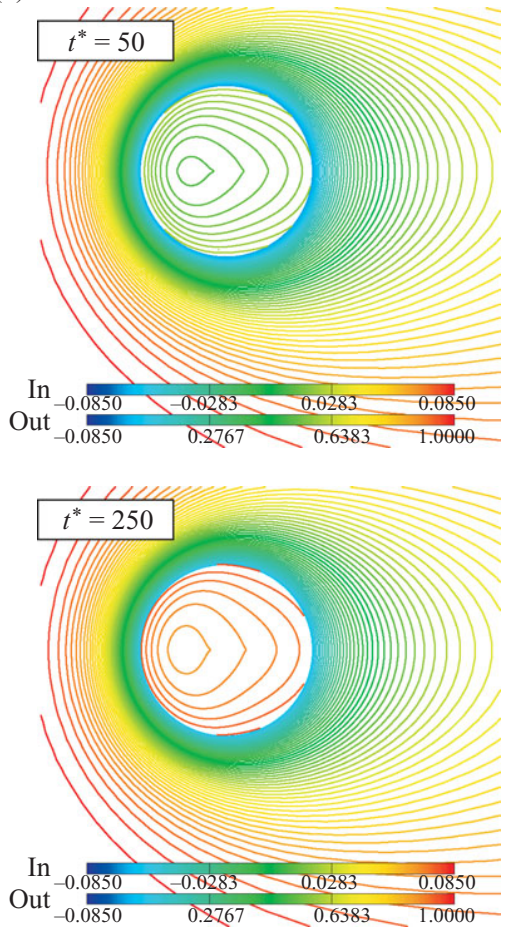

(b)
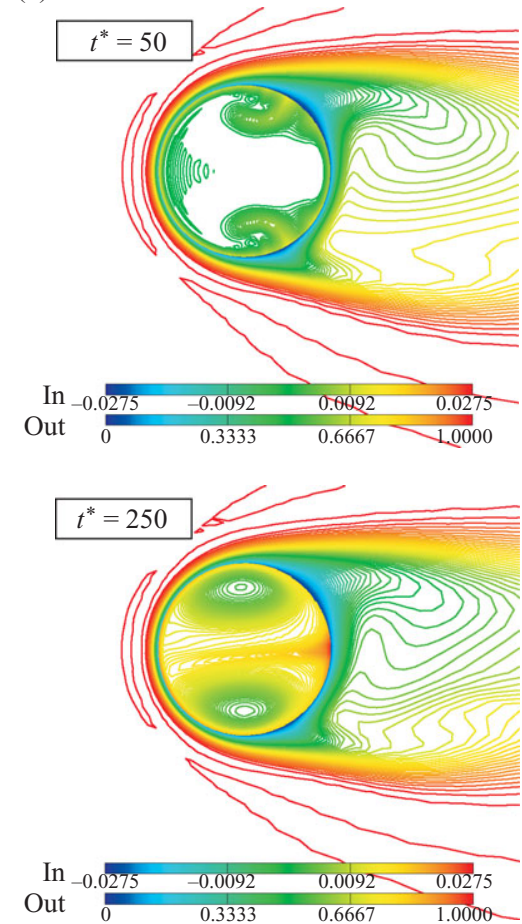

Figure 9. Time variations of distributions of instantaneous temperature $\Theta$ inside and outside a droplet on the $x-y$ plane for $H u=0.3$ : (a) $R e_{d}=10$ and (b) $R e_{d}=400$.

the droplet temperature is initially set to be lower than the ambient air temperature, it seems to be unreasonable for the droplet temperature to further decrease. In fact, Masoudi \& Sirignano (2000) showed by conducting the similar numerical simulations that the droplet temperature increases for a fuel droplet placed in a heated flow (the flow temperature is set to be several hundreds Kelvin higher than the droplet temperature). However, the decrease of the droplet temperature is considered to be due to the fact that the evaporative heat loss is higher than the convective heat gain from the higher temperature ambient air because the evaporation proceeds faster in the low-humidity condition. It is also observed that the droplet temperature for $R e_{d}=10$ decreases from the front of the droplet, whereas that for $R e_{d}=400$ decreases from the rear of the droplet since the cooled fluid in the vicinity of the droplet surface is transferred by the internal circulations.

The time variations of distributions of instantaneous temperature $\Theta$ inside and outside a droplet on the $x-y$ plane for the higher relative humidity of $H u=0.3$ are shown in figure 9. It is apparent that the temperature distribution inside the droplet is quite different from that for $H u=0$, although the temperature distribution outside the droplet is similar to $H u=0$. Namely, while the temperature inside the droplet decreases for $H u=0$, it increases for $H u=0.3$. This is because for $H u=0.3$ the evaporative heat loss is suppressed and then the convective heat gain from the higher temperature ambient air becomes greater, since the evaporation rate is drastically reduced by the higher relative humidity (see figure 4). 
Although the figures are omitted here, the Sherwood number $S h$ and Nusselt number $N u$ calculated for $R e_{d}=1,5,10,50,100,300$ and $400(H u=0$ and 0.3$)$ showed that these time-averaged values are not affected by the relative humidity and are in good agreement with the approximate expressions from previous experiments (Beard \& Pruppacher 1971).

\section{Conclusions}

A three-dimensional DNS was applied to flows inside and outside an evaporating spherical water droplet in air, and the effect of relative humidity on the heat transfer between the droplet and the ambient air was investigated. The initial air temperature was set to be $15 \mathrm{~K}$ higher than the initial droplet temperature. The main results obtained in this study can be summarized as follows:

(1) The local evaporative heat loss exhibits a maximum on the front of the droplet and decreases on going from the front to the rear, and the reduction on the rear becomes marked for high-droplet Reynolds numbers. This is because the evaporation rate is suppressed on the rear surface by the presence of flow separations, which make the vapour mass fraction gradient gentle in the vicinity of the droplet surface.

(2) The droplet evaporation rate and temperature are strongly affected by the relative humidity of air. The droplet temperature decreases in the low-humidity condition, whereas it increases in the high-humidity condition. The reason why the droplet temperature, which is initially lower than the ambient air temperature, decreases further in the low-humidity condition is that the low humidity enhances the droplet evaporation and causes the evaporative heat loss to be larger than the convective heat gain from the higher temperature ambient air at the droplet surface.

The authors would like to thank Professor Shigeru Takata of Kyoto University and Dr Ken-ichi Sugioka of Osaka Prefecture University for their useful discussion. They are also grateful to late Professor Yoshimoto Onishi of Tottori University for his many kind and useful suggestions on this work. This work was supported by Grant-in-Aid for Scientific Research (Nos. 19206023 and 20686015).

\section{REFERENCES}

BabA, Y. \& Kunose, R. 2008 Analysis and flamelet modeling for spray combustion. J. Fluid Mech. 612, 45-79.

BaGchi, P. \& Kottam, K. 2008 Effect of freestream isotropic turbulence on heat transfer from a sphere. Phys. Fluids 20, 073305.

Beard, K. V. \& Pruppacher, H. R. 1971 A wind tunnel investigation of the rate of evaporation of small water drops falling at terminal velocity in air. J. Atmos. Sci. 28, 1455-1464.

HANAZAKI, H. 1998 A numerical study of three-dimensional stratified flow past a sphere. J. Fluid Mech. 192, 393-419.

Kim, I., Elghobashi, S. E. \& Sirignano, W. A. 1995 Unsteady flow interactions between an advected cylindrical vortex tube and a spherical particle. J. Fluid Mech. 288, 123-155.

Kurose, R. \& Komori, S. 1999 Drag and lift forces on a rotating sphere in a linear shear flow. J. Fluid Mech. 384, 183-206.

Kurose, R., Makino, H., Komori, S., Nakamura, M., Akamatsu, F. \& Katsuki, M. 2003 Effects of outflow from surface of sphere on drag, shear lift, and scalar diffusion. Phys. Fluids 15, $2338-2351$.

Kurose, R., Misumi, R. \& Komori, S. 2001 Drag and lift forces acting on a spherical bubble in a linear shear flow. Intl J. Multiphase Flow 27, 1247-11258.

Masoudi, M. \& Sirignano, W. A. 2000 Collision of a vortex with a vaporizing droplet. Intl J. Multiphase Flow 26, 1925-1949. 
Effect of relative humidity on heat transfer across an evaporating water droplet 67

Miller, R. S. \& Bellan, J. 1999 Direct numerical simulation of a confined three-dimensional gas mixing layer with one evaporating hydrocarbon-droplet-laden stream. J. Fluid Mech. 384, 293-338.

MurRaY, F. W. 1967 On the computation of saturation vapor pressure. J. Appl. Meteorol. 6, 203-204.

Nakamura, M., Akamatsu, F., Kurose, R. \& Katsuki, M. 2005 Combustion mechanism of liquid fuel spray in a gaseous flame. Phys. Fluids 17, 123301.

ONISHI, Y. 1990 On the macroscopic boundary conditions at the interface for a vapour-gas mixture. In Adiabatic Waves in Liquid-Vapor Systems (ed. G. E. A. Meier \& P. A. Thompson), pp. 315-324. Springer.

SugioKa, K. \& Komori, S. 2005 The effects of droplets on mass transfer in wind-driven turbulence with breaking waves. Tran. JSPS (B) 71, 7-14 (in Japanese).

SugioKa, K. \& Komori, S. 2007 Drag and lift forces acting on a spherical water droplet in homogeneous linear shear air flow. J. Fluid Mech. 570, 155-175.

Welty, J. R., Wicks, C. E., Wilson, R. E. \& Rorrer, E. 1984 Fundamentals of Momentum, Heat, and Mass Transfer. John Wiley \& Sons. 